\title{
Cluster strong lensing in the Millennium Simulation: The effect of galaxies and structures along the line-of-sight
}

\author{
Ewald Puchwein ${ }^{1}$ and Stefan Hilbert ${ }^{2,1}$ \\ ${ }^{1}$ Max-Planck-Institut für Astrophysik, Karl-Schwarzschild-Straße 1, 85741 Garching, Germany \\ ${ }^{2}$ Argelander-Institut für Astronomie, Auf dem Hügel 71, 53121 Bonn, Germany
}

31 October 2018

\begin{abstract}
We use ray-tracing through the Millennium simulation to study how secondary matter structures along the line-of-sight and the stellar mass in galaxies affect strong cluster lensing, in particular the cross-section for giant arcs. Furthermore, we investigate the distribution of the cluster Einstein radii and the radial distribution of giant arcs. We find that additional structures along the line-of-sight increase the strong-lensing optical depth by $\sim 10-25 \%$, while strong-lensing cross-sections of individual clusters are frequently boosted by as much as $\sim 50 \%$. The enhancement is mainly due to structures that are not correlated with the lens. Cluster galaxies increase the strong-lensing optical depth by up to a factor of 2 , while interloping galaxies are not significant. We conclude that these effects need to be taken into account for predictions of the giant arc abundance, but they are not large enough to fully account for the reported discrepancy between predicted and observed abundances. Furthermore, we find that Einstein radii defined via the area enclosed by the critical curve are $10-30 \%$ larger than those defined via radial surface mass density profiles. The contributions of radial and tangential arcs to the radial distribution of arcs can be clearly distinguished. The radial distribution of tangential arcs is very broad and extends out to several Einstein radii. Thus, individual arcs are not well suited for constraining Einstein radii.
\end{abstract}

Key words: gravitational lensing - dark matter - large-scale structure of the Universe - galaxies: clusters: general - cosmology: theory - methods: numerical

\section{INTRODUCTION}

In studies of strong gravitational lensing, the mass distribution deflecting the light is typically assumed to be concentrated in a single region that is much smaller than the distance between source and observer. The extension of the matter distribution along the line-of-sight can be neglected in this case. The light deflection may then be described by projecting the lens' mass distribution onto a single plane and by assuming that light is deflected only when it passes this lens-plane and propagates along straight lines otherwise. In this so-called thin-lens approximation, the deflection angle is only sensitive to the projected surface mass distribution of the lens.

This approximation has been widely used to study many aspects of gravitational lensing. In particular, it was adopted to investigate the efficiency of galaxy clusters to form giant arcs. However, studies employing it together with spherically symmetric analytic cluster models (e.g. Wu and Mao 1996; Hattori et al. 1997; Molikawa et al. 1999) found significantly lower numbers of giant arcs than observed. The discrepancy is reduced when considering triaxially shaped halos (Oguri et al. 2003; Meneghetti et al. 2007). More re- alistic lens models that also contain substructure can be obtained from cosmological simulations. They were used by several authors to investigate the strong-lensing efficiency of clusters (see e.g. Bartelmann and Weiss 1994). One of the findings was that even when using such detailed numerical models, the number of observed giant arcs is significantly larger than the value expected in a $\Lambda \mathrm{CDM}$ cosmology (Bartelmann et al. 1998). The discrepancy can be reduced by taking cluster mergers (Torri et al. 2004), baryonic physics (Puchwein et al. 2005; Wambsganss et al. 2008; Rozo et al. 2008) and a broad source redshift distribution (Wambsganss et al. 2004; Li et al. 2005) into account. However, the question whether or not the number of observed giant arcs is compatible with a $\Lambda \mathrm{CDM}$ cosmology is still not settled, especially for a low value of the matter power spectrum normalisation $\sigma_{8}$ (Fedeli et al. 2008) as suggested by recent cosmological constraints (Komatsu et al. 2009).

Almost all of the studies mentioned above employed the thin-lens approximation and considered galaxy clusters as isolated gravitational lenses. In reality, however, clusters are embedded in a cosmic web of large-scale structures. In addition, other collapsed objects like galaxies or galaxy groups 
may be between a lensed background galaxy and the cluster or between the cluster and the observer and thus contribute to the lensing magnification and distortion. The effect of such additional structures along the line-of-sight has been ignored in most cluster-lensing studies.

A notable exception, in which the impact of such secondary structures was specifically investigated, is Wambsganss et al. (2005). Using a large cosmological simulation and multiple lens planes between source and observer, it was found that for high source redshift a significant fraction of the lines-of-sight with a convergence exceeding the critical value of unity for strong lensing/multiple image systems, does so only due to a contribution from a second subdominant lens-plane. This suggests that additional structures along the line-of-sight can, indeed, be important for strong lensing of high-redshift sources. From these results, it is, however, difficult to quantify the impact of such secondary structures on the abundance of giant arcs.

On the other hand, Hennawi et al. (2007) also used multiple lens planes in their analysis, but found that for sources at redshift $z_{\mathrm{s}}=2$, additional structures along the line-ofsight only mildly $(\leqslant 7 \%)$ affect the number of multipleimage systems with image separations larger than 10 arcsec. But then, the largest effect was found for even higher source redshift in Wambsganss et al. (2005) and there may be a significant contribution from smaller-separation strong-lensing systems.

There were also several studies investigating the impact of cluster galaxies on the lensing properties of clusters. Meneghetti et al. (2000) and Flores et al. (2000) found that the lensing efficiency of clusters is only mildly affected by its member galaxies. Brightest cluster galaxies, however, were not specifically accounted for in these studies, but were shown to be able to raise lensing cross-sections by $\sim 50 \%$ in Meneghetti et al. (2003). Dalal et al. (2004) showed that while the effect of galaxies on wide-separation giant arcs is small, it becomes significant for smaller $<15^{\prime \prime}$ separation arcs. Hilbert et al. (2008b) found that for point-like circular sources, galaxies can boost the number of images with length-to-width ratios exceeding 10 by up to a factor of 2 even when considering only images with separations $>5^{\prime \prime}$.

In this work we investigate in detail how strong cluster lensing is affected by secondary matter structures along the line-of-sight and by both cluster and interloping galaxies. We carefully quantify their impact and explore its dependence on cluster mass, cluster redshift, and source redshift. We also study how the total lensing efficiency of a realistic cluster population is affected. Our strong-lensing simulations employ ray-tracing along a backward light cone constructed from the Millennium simulation and use elliptical sources with sizes matching observations. The impact of galaxies is studied using a semi-analytic catalog that provides realistic galaxy properties and positions.

We also compare different definitions of the Einstein radius, as it is a practical quantity for characterizing the strength of a gravitational lens. Recently, Oguri and Blandford (2009) suggested to use the statistics of large Einstein radii as a cosmological probe, e.g. for measuring $\sigma_{8}$. Here, we present the Einstein radius distribution of the Millennium simulation clusters which can be used to calibrate such methods. Finally, we investigate at what cluster-centric radii giant arcs are most likely to be found.
The ray-tracing and strong-lensing simulation codes are introduced in Sect. 2, Our results are presented in Sect. 3 and summarized in Sect. 4 .

\section{METHODS}

\subsection{Ray-tracing}

We employ ray-tracing through the Millennium Simulation (Springel et al. 2005), a large $N$-body simulation of cosmic structure formation, to study the light deflection by matter structures in the observer's backward light cone. The Millennium Simulation assumes a flat $\Lambda$ CDM cosmology with a matter density $\Omega_{\mathrm{M}}=0.25$ (in units of the critical density), a cosmological constant with energy density $\Omega_{\Lambda}=0.75$, a Hubble constant of $h=0.73$ (in units of $100 \mathrm{~km} \mathrm{~s}^{-1} \mathrm{Mpc}^{-1}$ ), and a scale-invariant initial density power spectrum with normalisation $\sigma_{8}=0.9$. The simulation used a TreePM algorithm (Springel 2005) with $10^{10}$ particles of mass $m_{\mathrm{p}}=8.6 \times 10^{8} h^{-1} \mathrm{M}_{\odot}$ and a force softening of $5 h^{-1} \mathrm{kpc}$ comoving to simulate the structure formation in a cubic region of $L=500 h^{-1} \mathrm{Mpc}$ comoving side length. Simulation snapshots were stored on disk at 64 output times between redshift $z=127$ and $z=0$.

We use the multiple-lens-plane algorithm described in Hilbert et al. (2008a) to calculate the gravitational deflection of light by matter inhomogeneities between the source and the observer. The observer's backward light cone is divided into redshift slices. The matter content of each slice is then projected onto a lens plane transverse to the lineof-sight. Light rays are traced back from the observer to their source under the assumption that photons propagate unperturbed between these lens planes, but photons passing through a plane are deflected by an amount that is determined by the projected matter distribution on the plane.

The distribution of the dark matter in the observer's light cone is generated directly from the particle data of the Millennium Simulation. We use one redshift slice for each simulation snapshot. Each slice is filled with the simulation particles of the corresponding snapshot. The particles are projected onto a hierarchy of meshes on the corresponding lens plane. The matter distribution on the meshes is smoothed by an adaptive scheme that retains a resolution of about $5 h^{-1} \mathrm{kpc}$ comoving in dense regions (e.g. in the centres of massive dark-matter halos). The light deflection due to the dark matter is then calculated from the smoothed distribution with Fast-Fourier-Transform methods, finite differencing, and bi-linear interpolation.

The distribution of the luminous matter in the light cone is inferred from the semi-analytic galaxy-formation model by De Lucia and Blaizot (2007). The galaxies within each redshift slice are projected onto the same lens plane as the simulation particles. For each galaxy, the projected stellar matter is assumed to follow a de-Vaucouleurs profile (de Vaucouleurs 1948) for the bulge component, and an exponential surface density profile for the disc component. The light deflections induced by stars in the galaxies are then calculated by analytic expressions 1

1 Details about the treatment of the light deflection by the stellar matter can be found in Hilbert et al. (2008b). 
In order to study the lensing properties of galaxy groups and clusters, we select a sample of massive dark-matter halos at three lens redshifts $z_{\mathrm{L}}=0.28,0.62$, and 0.99 in the Millennium Simulation. For each halo, we set up light rays starting from the observer with directions on a regular grid of $1024 \times 1024$ rays in a $6^{\prime} \times 6^{\prime}$ field-of-view. The observer position is chosen such that the halo centre is contained in the light cone spanned by the rays. The rays are traced back through the series of lens planes, and the ray positions on each lens plane, which also serve as source planes, are recorded. These ray positions are then used to create lensed galaxy images as described in Sect. 2.2.

In this work, we want to investigate the influence of the stellar mass in galaxies on the strong-lensing properties of groups and clusters. We thus perform the ray-tracing not only as described above, but also by ignoring the contribution from the stellar matter in galaxies to the light deflection. We then compare the results we obtain by either including or ignoring light deflection by the luminous matter.

Furthermore we want to quantify the contribution from additional matter structures along the line-of-sight. Therefore in addition to the complete multiple-lens-plane raytracing (hereafter full ray-tracing), we also perform raytracing in the following ways: In one set of ray-tracing simulations, we only take into account the light deflection by the lens plane containing the selected dark-matter halo (singleplane ray-tracing). These ray-tracing simulations still include the effects of correlated structures close to the lensing cluster but neglect contributions from matter structures at different redshifts. In addition we perform another set of simulations, in which only the matter in a cubic region of $4 h^{-1} \mathrm{Mpc}$ comoving side length around the halo centre is taken into account in the ray-tracing (cluster-only raytracing).

\subsection{Strong-lensing simulations}

We investigate strong lensing in these simulated light cones by placing sources on six different source planes at redshifts $z_{\mathrm{S}}=1.0,1.5,2.1,3.1,4.2$, and 5.7. We then find the images of each source using the ray positions from the ray-tracing described above and calculate the length $L$, width $W$ and magnification for each image. This allows a statistical characterisation of the strong-lensing properties of the matter structures in each light cone.

For finding the images of a large number of source galaxies, we follow the method introduced by Miralda-Escude (1993a,b) and adapted to non-analytic models by Bartelmann and Weiss (1994) and Bartelmann et al. (1995). The algorithm is described in some detail in Puchwein et al. (2005). A previous version is also discussed in Meneghetti et al. (2000).

First, the critical curves and caustics for each lens are determined by checking where the determinant of the Jacobian of the lens mapping changes its sign. Then, the algorithm adaptively places source galaxies on the source plane such that regions near caustics are sampled with a higher source density than regions far from caustics. A statistical weight for each source accounts for the varying source density. Source galaxies are modelled as ellipses with equivalent radii of $0.85,0.65,0.45,0.3,0.25$, and 0.25 arcsec at redshifts
$z_{\mathrm{S}}=1.0,1.5,2.1,3.1,4.2$, and 5.7 respectively 2 Source ellipticities are assigned by randomly drawing ratios of major to minor axis from the interval [1,2].

Each of the ray-traced fields-of-view is covered by a grid of $4096 \times 4096$ pixels in the image plane. The source-plane positions of these pixels are calculated by bi-linear interpolation between the source-plane positions of the $1024 \times 1024$ rays that were traced through the simulation as described in Sect. 2.1 All image pixels of a source are then found by checking which of the grid pixels correspond to a sourceplane position that is enclosed by the ellipse representing the considered source galaxy. The image pixels are then grouped into images.

The properties of an image are obtained by finding the image pixel $p_{1}$ that falls closest to the source centre when mapped to the source plane, the pixel $\mathrm{p}_{2}$ that is the farthest from $\mathrm{p}_{1}$, and the pixel $\mathrm{p}_{3}$ that is the farthest from $\mathrm{p}_{2}$. We then fit a circle through these three points and use the arc length from $\mathrm{p}_{2}$ to $\mathrm{p}_{3}$ as the length of the image. The image area is calculated directly from the number of image pixels, while its perimeter is obtained by walking along the boundary pixels and summing up their mutual distances. We apply the same resolution corrections to image length and perimeter as in Puchwein et al. (2005), although they are less important here due to the better image plane grid resolution 3 We then compute a simple geometric figure (ellipse, circle, rectangle or ring) with equal area and length to determine the image width $W$, which is approximated by the minor axis of the ellipse, the diameter of the circle, the smaller side of the rectangle or the width of the ring, respectively. We choose the type of the figure by comparing its circumference to the perimeter of the image.

Finally, for all light cones and each source redshift, we quantify the efficiency to produce arcs, which we define as images with a length-to-width ratio $L / W>7.5$. More precisely, we determine strong-lensing cross-sections $\sigma_{L / W>7.5}$ by summing up the statistical weights of the sources that have images with $L / W>7.5$ and calculating the corresponding area in the source plane. If there is more than one arc for a source, we multiply the statistical weight of this source by the number of arcs.

\section{RESULTS}

In order to quantify the impact of additional matter structures along the line-of-sight on strong cluster lensing, we selected in total 100 cluster-sized halos, only by their mass, from the Millennium simulation. Table 1 summarizes their distribution in redshift $z_{\mathrm{L}}$ and halo mass $M_{200}^{\text {crit }} 4$ For each

\footnotetext{
2 These source radii agree well with measured half-light radii of galaxies at these redshifts (Ferguson et al. 2004).

3 We also performed several strong lensing simulations with an increased $8192 \times 8192$ grid resolution to check that results are robust even for the smallest source sizes. In all cases, strong lensing cross sections changed by less than $10 \%$ compared to the lower resolution results. Also note that any small residual resolution dependence will affect the results obtained by different kinds of ray-tracing in the same way.

4 Throughout this paper, we define cluster mass as the mass within a spherical region with a mean density 200 times the critical density of the universe at the cluster redshift.
} 


\begin{tabular}{lccccc}
\hline mass bin & $(\mathrm{a})$ & $(\mathrm{b})$ & $(\mathrm{c})$ & $(\mathrm{d})$ & $(\mathrm{e})$ \\
$M_{200}^{\text {crit }}\left[10^{14} h^{-1} \mathrm{M}_{\odot}\right]$ & $1-2$ & $2-4$ & $4-8$ & $8-16$ & $>16$ \\
\hline \#clusters in bin & & & & & \\
at $z_{\mathrm{L}}=0.28$ & 10 & 10 & 10 & 8 & 1 \\
at $z_{\mathrm{L}}=0.62$ & 10 & 10 & 10 & 4 & 0 \\
at $z_{\mathrm{L}}=0.99$ & 10 & 10 & 7 & 0 & 0 \\
\hline
\end{tabular}

Table 1. Mass range and number of studied clusters within the mass bins at redshifts $z_{\mathrm{L}}=0.28,0.62$, and 0.99 . We aim to use 10 clusters per bin, but there are $<10$ clusters in the Millennium simulation in the mass range of some bins. Also within each bin, clusters are roughly constantly spaced in logarithmic cluster mass.

cluster, we then performed full, single-plane, and clusteronly ray-tracing simulations, as described in Sect. 2.1 and calculated the corresponding strong-lensing cross-sections as detailed in Sect. 2.2. Comparing them to each other allows us to clearly pin down the effect of additional line-of-sight structures on cluster lensing properties and giant arc statistics.

For all selected clusters, we also performed full and cluster-only ray-tracing simulations that ignored the light deflection by the stellar matter in both cluster and interloping galaxies. This enables us to also quantify the impact of the stellar component of galaxies on strong cluster lensing.

\subsection{The impact of additional structures along the line-of-sight}

In Fig. 1. we show the average strong-lensing cross-sections $\sigma_{L / W}>7.5$ for all cluster mass bins given in Table 1 and all considered source and lens redshifts. For each mass bin and lens redshift, a set of three curves illustrates the results obtained when using the full, single-plane, and cluster-only ray-tracing. In all cases, the stellar matter in galaxies was accounted for in the ray-tracing. In the left panels, all suitable arcs in the whole $6^{\prime} \times 6^{\prime}$ ray-tracing fields where considered for calculating $\sigma_{L / W>7.5}$.

Probably the most obvious result is that the average cross-section is a strongly increasing function of source redshift for all mass bins, lens redshifts, and types of raytracing. Clusters are, thus, more efficient in lensing source galaxies at higher redshift. This finding is in good agreement with previous studies (Wambsganss et al. 2004; Li et al. 2005; Hilbert et al. 2007).

A more careful inspection of the results reveals that for all considered lens redshifts, the cross-sections of the most massive clusters (uppermost set of curves in each panel) are only very mildly affected by additional matter structures along the line-of-sight. The results obtained using full raytracing are very similar to those found by single-plane and cluster-only ray-tracing for such massive clusters.

The effects of additional matter structures along the line-of-sight are more important for less massive clusters and for sources at high redshift. There, the cross-sections obtained when using the complete multiple-lens plane raytracing are on average $\sim 20-40 \%$ larger than those found by considering only light deflection by the cluster. On the other hand, the difference between results from single-plane and cluster-only ray-tracing are still very small. Thus the increase in lensing efficiency found when accounting for all the matter in the light cone is not due to correlated structures near the cluster, but due to uncorrelated structures along the line-of-sight.

So far, all suitable arcs, i.e. with a length-to-width ratio exceeding 7.5, found in the ray-traced field were counted for the computation of a cluster's strong-lensing cross-section. However, an observer might not assign all these arcs to the selected cluster, but also to other objects that happen to be in the ray-traced field. Neglecting this is unlikely to significantly bias results for the most massive clusters. However, the smallest clusters in our sample are in some cases not even the most prominent lens in the field-of-view. Thus by assigning all found arcs to such a cluster, one would overpredict its lensing efficiency, especially when using the full ray-tracing.

To prevent such misassignments from biasing our results, we repeated the computation of $\sigma_{L / W}>7.5$ for all cluster, but this time counting only arcs within 5 Einstein radii from the selected cluster's centre. Note that here, we define the Einstein radius $\theta_{E}$ by

$$
\theta_{E}=\sqrt{\frac{A}{\pi}}
$$

where $A$ is the solid angle enclosed by the cluster's outer critical curve and calculated using cluster-only ray-tracing. In this way, we largely avoid assignments to a wrong object. In rare cases of ongoing mergers or very elliptic clusters with substructure, we might, however, miss some arcs that are very distant from the cluster centre but should probably be assigned to it. We employ this cut in image separation troughout the remainder of this paper.

Results obtained in this way are shown in the right panels of Fig. 1. As expected, the cross-sections of the most massive clusters are almost unchanged. For the smallest clusters on the other hand, the lensing cross-section obtained by full ray-tracing is reduced and becomes more similar to the cluster-only results. Thus, when counting only arcs within 5 Einstein radii, most small clusters are not significantly affected by additional structures along the line-of-sight. The effect is biggest for intermediate mass clusters, where crosssections increase on average by roughly $\sim 15-30 \%$.

For individual clusters, however, effects can be significantly larger. We illustrate this in Fig. 2 which shows the ratio of the cross-section obtained by the full ray-tracing to the values found by cluster-only ray-tracing. For the sake of clarity, only the results for the $z_{\mathrm{L}}=0.28$ clusters and three different source redshifts are shown. Again, only arcs within 5 Einstein radii were counted. Boosts of the cross-sections by additional matter structures of up to $50 \%$ happen frequently. There are also a couple of clusters with significantly larger ratios. For most of them, however, absolute values of the cross-sections are quite small, so that a small absolute increase results in a large relative boost.

A particularly impressive example is the second most massive cluster shown in the figure. In spite of its enormous mass, secondary structures along the line-of-sight boost its cross-section for lensing high-redshift sources by roughly $60 \%$. A large part of this increase is due to three group-sized halos, two at redshift 1.5 and one at redshift 1.63 , which are close to the cluster in projection. In addition, there is a significant effect due to a $2 \times 10^{12} \mathrm{M}_{\odot} / h$ halo that is also at redshift 1.63. Normally, one would not expect such a small 


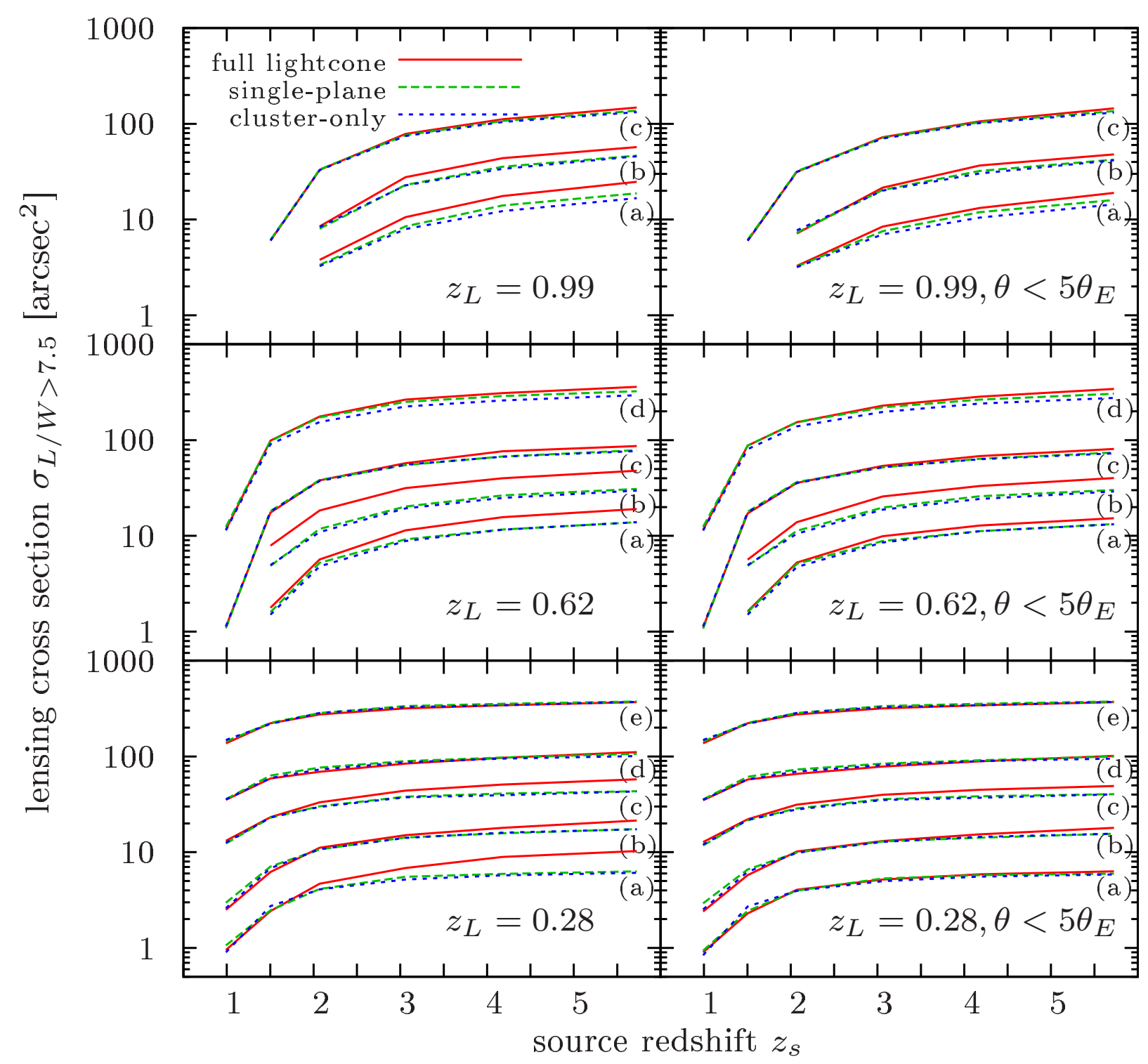

Figure 1. Average strong-lensing cross-sections $\sigma_{L / W>7.5}$ of clusters in mass bins (a)-(e) (see Table 1). The cross-sections were obtained using full light cones (red solid lines), only one lens plane containing the selected cluster (green long-dashed), and light deflection only by the selected cluster (blue short-dashed). Results for clusters at redshift $z_{\mathrm{L}}=0.28$ (bottom panels), $z_{\mathrm{L}}=0.62$ (middle panels), and $z_{\mathrm{L}}=0.99$ (top panels) are shown. In the left panels, arcs in the whole $6^{\prime} \times 6^{\prime}$ field were considered, while in the right panels, only arcs within 5 Einstein radii were included.

object to have a noticeable impact on the lensing properties of a cluster whose mass is almost three orders of magnitude larger. This halo is, however, itself strongly lensed by the foreground galaxy cluster. The peak in the lensing convergence corresponding to it is shaped like a long thin tangential arc. Due to the lensing magnification of this halo, its light deflection affects a much larger number of lines-of-sight. In this way, this small object can significantly alter the critical curve of the cluster. Also see Hilbert et al. (2008a) for a discussion of how multiple-lens-plane ray-tracing can be used to study lens-lens coupling effects in weak lensing. Finally, there is also some contribution from several halos with masses of a few $10^{12} \mathrm{M}_{\odot} / h$ and redshifts between $z_{\mathrm{L}}=1.7$ and 3.3. Remarkably, the importance of additional line-ofsight structures is already strongly reduced for sources at a somewhat lower redshift of $z_{\mathrm{s}}=3.1$. This is because the efficiencies of lenses at $z_{\mathrm{L}}>1.5$ significantly increase with source redshift between $z_{\mathrm{s}}=3.1$ and 5.7 , while there is only a marginal gain for a lens at the much lower cluster redshift of $z_{\mathrm{L}}=0.28$.

There are also several clusters which have a smaller cross-section when including the effects of additional structures along the line-of-sight. However, the decrease is less than $10 \%$ in most cases. Note that such clusters are not necessarily projected onto underdense regions, as we do not find a clear correlation between change in cross-section and change in average convergence within 5 Einstein radii 5 In

5 The region inside which the average convergence was computed 


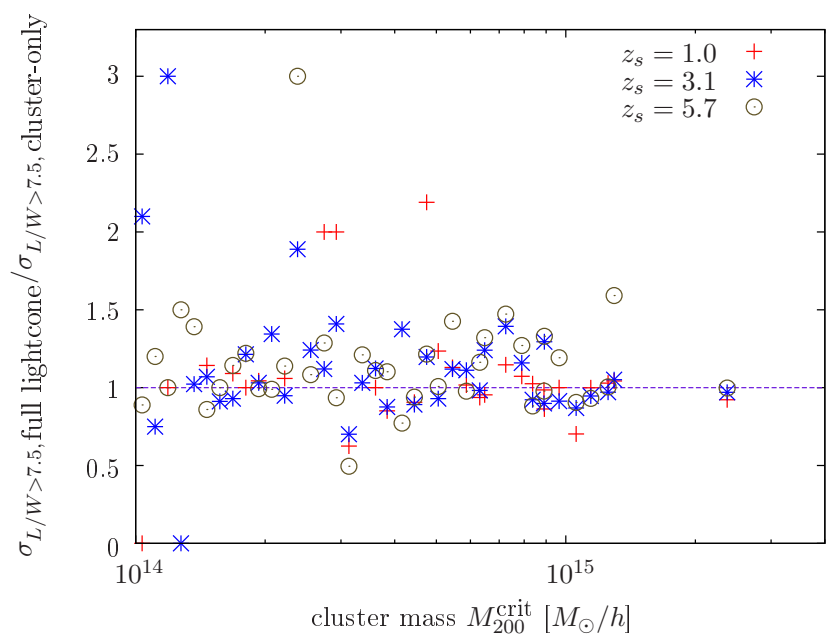

Figure 2. Ratio of the strong-lensing cross-section $\sigma_{L / W}>7.5$ found when using the full multiple-lens-plane ray-tracing to the value obtained when considering only light deflection by the selected cluster. Results are shown as a function of cluster mass for all $z_{\mathrm{L}}=0.28$ clusters in our sample. Three different source redshifts, $z_{\mathrm{S}}=1.0,3.1$, and 5.7 were used. Only arcs within 5 Einstein radii were included in the analysis.

most cases where we find significantly smaller cross-sections when including all structures in the light cone, it is the 'external' shear caused by the secondary structures along the line-of-sight that counteracts the cluster shear on potential arcs. In many cases, this lowers their length-to-width ratios below the adopted threshold value of 7.5.

Figure 3 shows the critical curves and caustics of two clusters and illustrates how they change when the effects of additional structures along the line-of-sight are included. The first cluster, shown in the figure's upper panels, is an example of a strongly affected object. Its lensing cross-section is boosted by a factor of two, and the shape of its critical curve changes significantly. The latter is stretched and merges with the critical curve of a subhalo. Also shown are the centres of all giant arcs formed in the cluster's full and cluster-only strong-lensing simulations as well as the sources that give rise to them. They illustrate how the shape of the cluster's strong-lensing cross-section changes and where additional arcs appear. The second cluster is a more typical example. Its cross-section increases by about $\sim 30 \%$, mostly due to an additional structure below and to the right of the cluster. The shape of its critical curve and caustic are only mildly affected. One can however clearly see that the caustics are shifted with respect to each other due to light deflection by structures between the cluster and the source plane.

As mentioned above and illustrated in Fig. 1, the impact of additional structures along the line-of-sight on cluster strong-lensing cross-sections depends on cluster mass. Thus, one needs to take the steep cluster mass function into account when investigating how the total lensing efficiency of a realistic cluster population is affected. However, instead of employing an analytic mass function for that purpose, we

was kept fixed, as we used the Einstein radius obtained from cluster-only ray-tracing in both cases. directly use the full Millennium simulation halo catalogue. We start from the cross-sections we calculated for our selected cluster sample and then assign a cross-section to all other Millennium simulation clusters, here defined as halos with $M_{200}^{\text {crit }}>10^{14} h^{-1} \mathrm{M}_{\odot}$, by interpolating linearly in cluster mass. Summing over all clusters finally yields an estimate of the total strong-lensing cross-section of the Millennium simulation cluster population at $z_{\mathrm{L}}=0.28,0.62$, and 0.99 . We calculated these quantities based both on full and on cluster-only ray-tracing. Figure 4 illustrates how additional structures along the line-of-sight affect the lensing efficiency of the cluster population as a whole and thus the contribution of clusters at these redshifts to the strong-lensing optical depth. Clearly, the lensing efficiency for high-redshift sources increases when taking such additional structures into account. This effect is larger for intermediate and high redshift cluster populations, where we find boosts up to $30 \%$. For the $z_{\mathrm{L}}=0.28$ clusters and high redshift sources, the lensing efficiency increases by about $10 \%$.

We also show $68 \%$ confidence intervals of the total crosssection ratios. For each cluster redshift, they were calculated from $10^{4}$ bootstrap cluster samples. Note that we bootstrap both the clusters which we use for the cross-section interpolation as well as the full cluster population in the simulation. The former accounts for uncertainties due to the limited number of objects for which we have performed stronglensing simulations and for noise in the cross-section calculation, while the latter accounts for cosmic variance in the whole simulation 6 Most uncertain is the contribution from the large number of low mass clusters to the strong-lensing optical depth. This is because their cross-sections are small, especially for low source redshift, and thus the relative error in their determination is larger.

Of course, the strong-lensing cross-section of individual clusters does not only depend on cluster mass, but due to halo triaxiality, also on orientation (Dalal et al. 2004), as well as on halo concentration (see e.g. Hennawi et al. 2007), and dynamical state (Torri et al. 2004). It is, therefore, not suprising that the distribution of cross-sections is quiet broad even at constant cluster mass. Obviously, the impact of structures along the line-of-sight also depends on the line-of-sight's orientation and can change significantly depending on it. Thus, while the curves given for individual mass bins in Fig. 1 (and Fig. [5 in the next section) are very useful for identifying trends with cluster mass, they may, due to the limited number $(\leqslant 10)$ of objects per bin, not always be fully representative for all cluster lenses in that mass range. The results we give for the whole cluster population, on the other hand, should be much more robust as a larger number of clusters is used for their derivation. Also note that the bootstrap error estimates include the effects mentioned above.

6 Before we applied bootstrapping, we have performed a MonteCarlo analysis that employed random cross-sections drawn based on a rough estimate of the cross-section cluster mass relation and its scatter to confirm that bootstrapping gives reliable error estimates even when using such an interpolation scheme to calculate total cross-section ratios. 


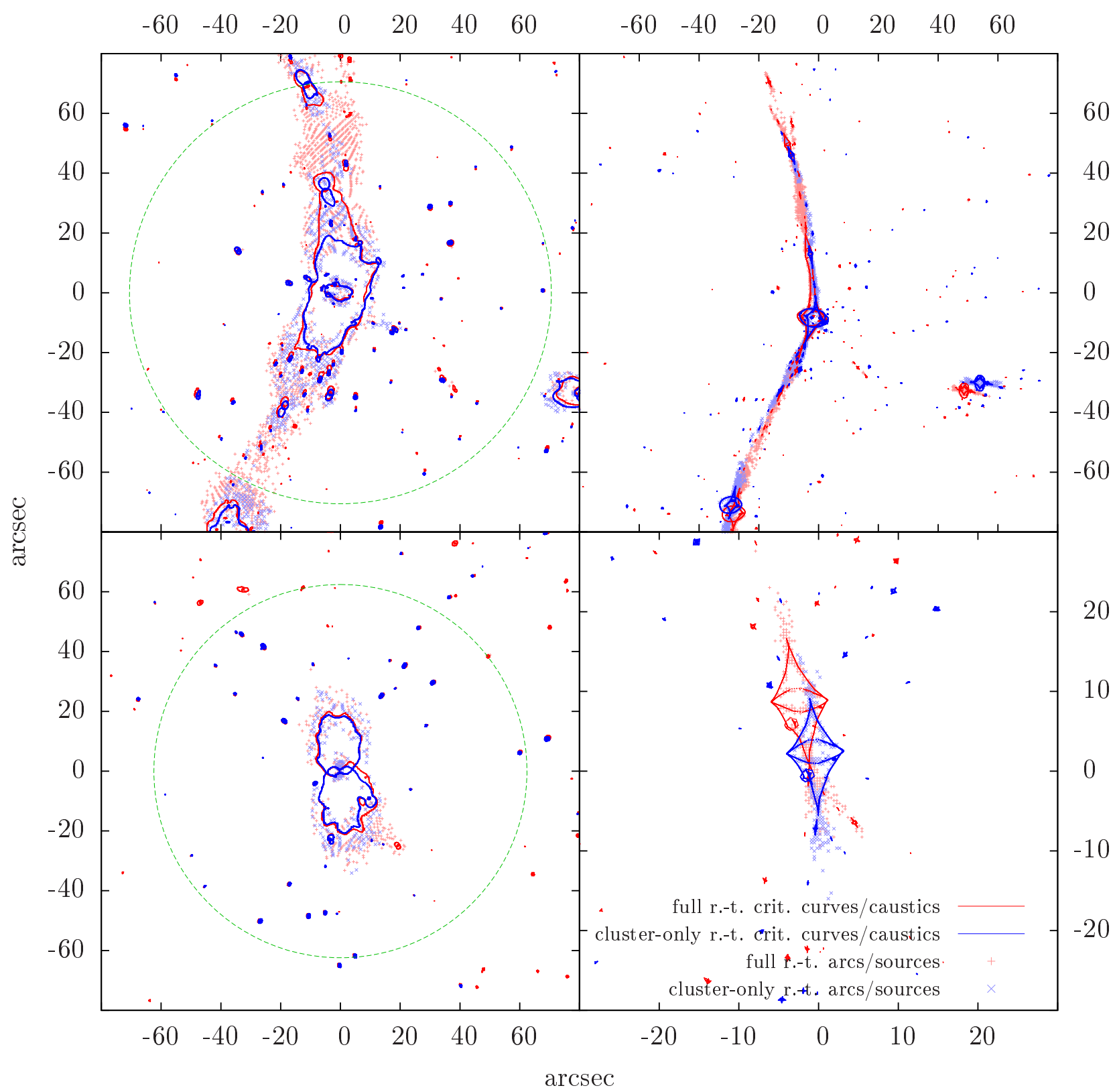

Figure 3. Critical curves (left panels) and caustics (right panels) of a $10^{15} h^{-1} \mathrm{M}_{\odot}$ cluster at $z_{\mathrm{L}}=0.62$ (upper panels) and a $7 \times$ $10^{14} h^{-1} \mathrm{M}_{\odot}$ cluster at $z_{\mathrm{L}}=0.28$ (lower panels). Curves obtained by considering light-deflection only by the cluster (blue) as well as by all matter structures along the line-of-sight $(r e d)$ are shown. A source redshift of $z_{\mathrm{S}}=3.1$ was assumed. The cluster shown in the upper panels is a prominent example for an object whose lensing efficiency is strongly boosted by additional structures along the line-of-sight. Its cross-section increases by almost a factor of 2 . The lower panels show a more typical example, where the cross-section increases by $\sim 30 \%$. In both cases only giant arcs within 5 Einstein radii (green circles) were counted. In the left panels, the centres (defined by pixel $\mathrm{p}_{1}$ as described in Sect. 2.2) of all arcs with $L / W>7.5$ found in the strong-lensing simulation are shown. In the right panels, the positions of the sources that give rise to these arcs are indicated. Note that for the sake of clarity, we choose a different scale in the bottom, right panel.

\subsection{The impact of the stellar mass in galaxies}

In Fig. 5, we compare the strong-lensing cross-sections calculated accounting for the light deflection by the stellar component of galaxies to those found ignoring all stellar matter. Average cross-sections for all the $z_{\mathrm{L}}=0.28,0.62$ and 0.99 cluster mass bins listed in Table 1 are shown. Results obtained by both full and cluster-only ray-tracing are indicated. When accounting for stellar matter, the former in- clude the effects of all galaxies between source plane and observer while the latter include only the effects of cluster galaxies. One can clearly see that the stellar matter significantly increases the strong-lensing cross-sections of clusters, especially for low-mass systems and for low source redshift. In other words, the effect is largest for the most inefficient lenses, while very massive clusters are only mildly affected. It is also worth noting that the impact of stellar matter is very similar when using full and cluster-only ray-tracing. 


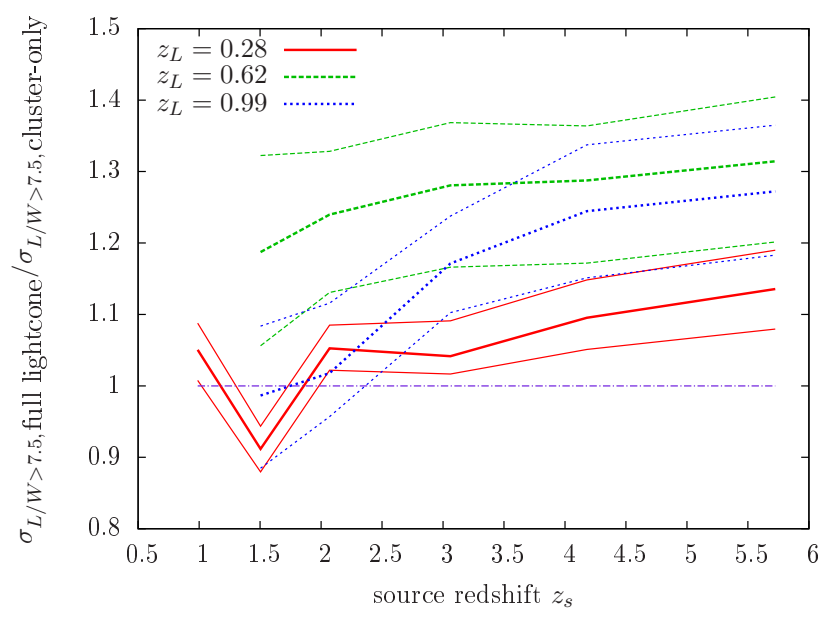

Figure 4. Impact of additional structures along the line-of-sight on the combined strong-lensing cross-section of all Millennium simulation clusters. Ratios of total cross-sections estimated from full and cluster-only ray-tracing are shown as a function of source redshift for the cluster populations at $z_{\mathrm{L}}=0.28,0.62$, and 0.99 (thick lines). Only arcs within 5 Einstein radii were included in the analysis. Also shown are $68 \%$ confidence intervals of the ratios (thin lines) obtained by bootstrapping techniques.

This means that most of the increase is due to the stellar component of cluster galaxies, while interloping galaxies between source and cluster or between cluster and observer play only a minor role.

Also in this context, the impact on cluster lensing cross-sections depends on cluster mass. We, thus, use the same method as in Sect. 3.1 to estimate how the lensing efficiency of a realistic cluster population is affected. Figure 6 illustrates the increase of the combined stronglensing cross-section of all Millennium simulation clusters with $M_{200}^{\text {crit }}>10^{14} h^{-1} \mathrm{M}_{\odot}$. Relative changes due to the stellar components of galaxies are shown as a function of source redshift for all clusters at $z_{\mathrm{L}}=0.28,0.62$ and 0.99 . We also plotted the $68 \%$ confidence intervals of the cross-section ratios obtained by a bootstrap error analysis similar to that described in Sect. 3.1 Full ray-tracing was used to derive all the data shown in the figure. Stellar matter boosts the lensing efficiency of the $z_{\mathrm{L}}=0.28$ cluster population by almost a factor of two for low redshift sources. For high-redshift sources the strong-lensing efficiency still increases by about $50 \%$. The clusters at higher redshift show the same trend in source redshift with somewhat larger boosts for low redshift sources. However, the magnitude of the effect is only about half of that found for the $z_{\mathrm{L}}=0.28$ clusters.

In order to understand this difference better, we calculated the Einstein radius of the average strong-lensing cluster for all lens redshifts. More, precisely we assigned an Einstein radius to all Millennium simulation clusters using the same interpolation method as for the cross-sections. We then derived the cross-section-weighted Einstein radii for the three cluster populations and all source redshifts. We found that for sources at redshift 1.5 the Einstein radius of the average lensing cluster is very similar for clusters at $z_{\mathrm{L}}=0.28$ and 0.62 , namely about $\sim 8$ arcsec, and larger in the latter case for higher redshift sources. For the $z_{\mathrm{L}}=0.99$ clusters it is compareable or larger than for the $z_{\mathrm{L}}=0.28$ clusters

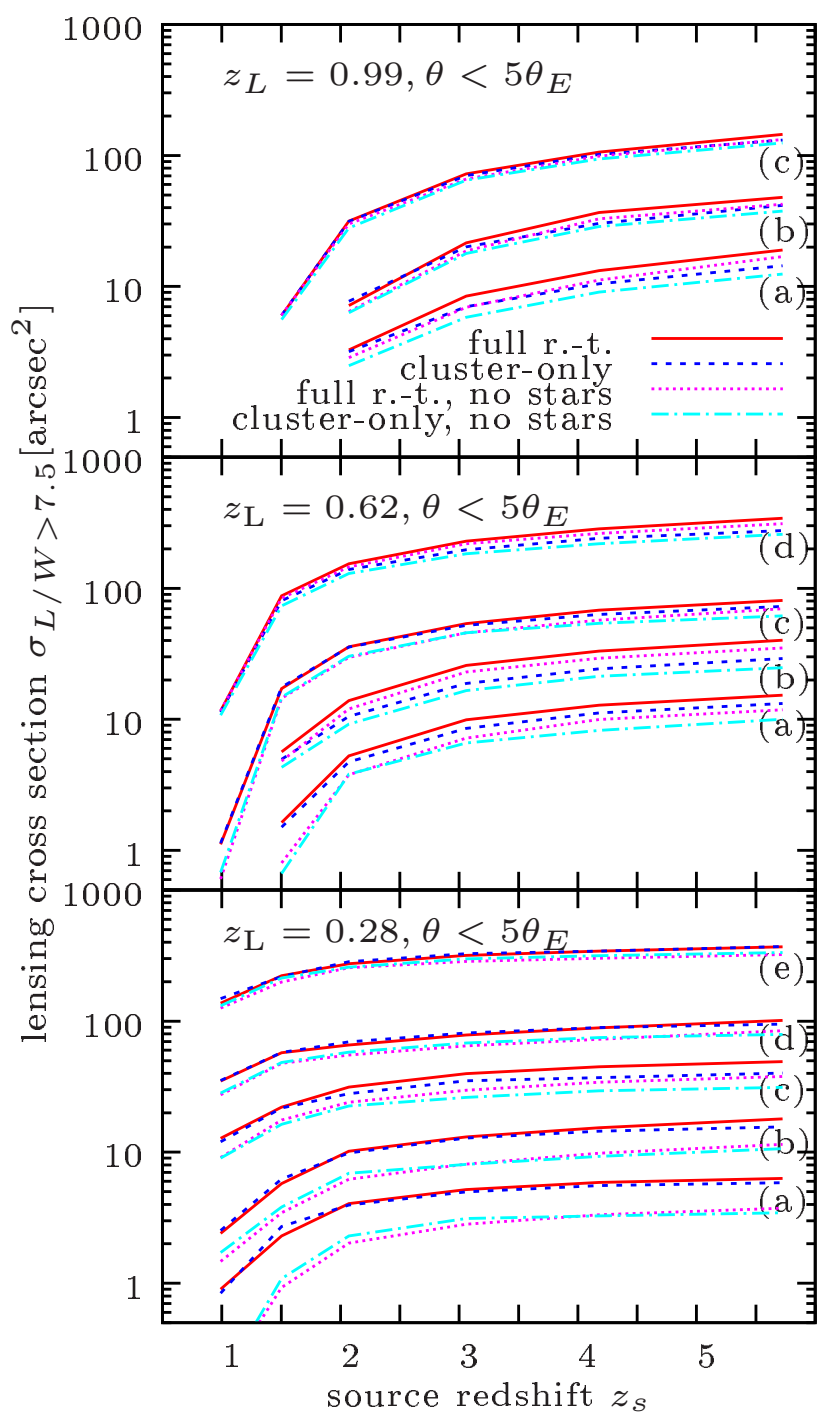

Figure 5. Average strong-lensing cross-sections $\sigma_{L / W>7.5}$ of clusters in mass bins (a)-(e) at cluster redshift $z_{\mathrm{L}}=0.28$ (bottom panel), $z_{\mathrm{L}}=0.62$ (middle panel), and $z_{\mathrm{L}}=0.99$ (top panel). The cross-sections were obtained using full ray-tracing and accounting for stellar matter (red solid lines), cluster-only ray-tracing and accounting for stellar matter (dark-blue, dashed), full ray-tracing and ignoring stellar matter (magenta, dotted), cluster-only raytracing and ignoring stellar matter (light-blue, dot-dashed). Only arcs within 5 Einstein radii were counted. The red solid and darkblue dashed curves show the same data as in the middle right and lower right panels of Fig. 1

above a source redshift of 2.1. Thus, in spite of the much larger distance between lens and observer the Einstein angle of strong-lensing selected clusters does not decrease at high lens redshift. It corresponds, however, to a larger physical distance at the cluster position. The stellar mass within the critical curve is typically dominated by the central galaxy, which is more concentrated than the dark matter halo. Thus, when the physical size of the critical curve increases lensing becomes more dark matter dominated and less affected by stellar matter.

Our results for the increase of the lensing cross sections due to cluster galaxies are in good agreement with 


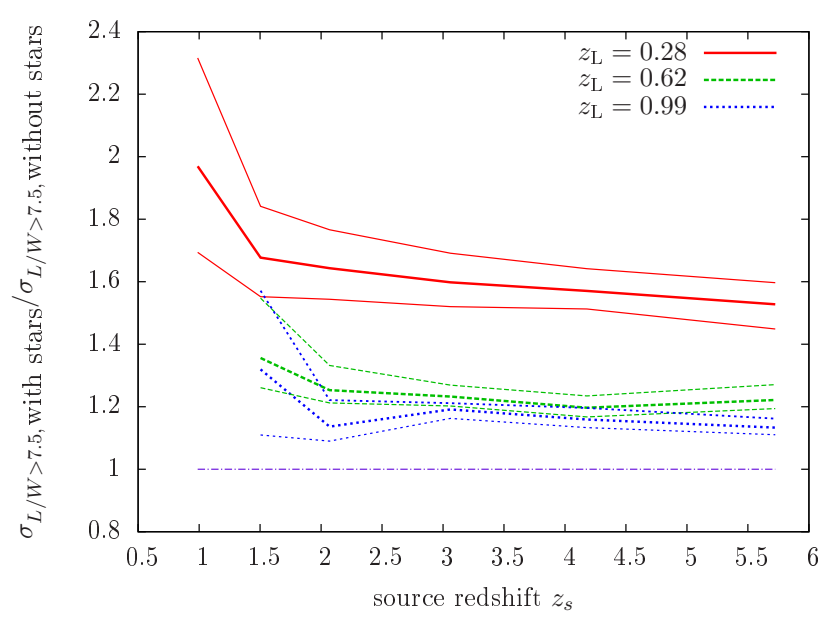

Figure 6. Impact of stellar matter on the combined stronglensing cross-section of all Millennium simulation clusters. Ratios of the total cross-sections estimated accounting for and ignoring stellar matter are shown as a function of source redshift for the cluster populations at $z_{\mathrm{L}}=0.28,0.62$, and 0.99 (thick lines). Also shown are $68 \%$ confidence intervals of the ratios (thin lines) obtained by bootstrapping techniques. The analysis employed full ray-tracing. Only arcs within 5 Einstein radii were included.

the results of Hilbert et al. (2008b) for point-like sources. At first glance, these results may seem to be at odds with the findings of Meneghetti et al. (2000). However, we find the largest effect in small clusters which were not considered there. In addition, we also accounted for brightest cluster galaxies which were also not included in that study, but shown to be able to raise lensing cross-sections by $\sim 50 \%$ in Meneghetti et al. (2003).

\subsection{Optical depths for giant arcs}

We can use our cluster sample to estimate the optical depth $\tau_{L / W>7.5}$ for arcs with $L / W>7.5$ as a function of source redshift $z_{\mathrm{S}}$. For the considered cluster lens redshifts $z_{\mathrm{L}}=0.28,0.62$, and 0.99 , we calculate the crosssections $\sigma_{L / W>7.5, i}$ of every halo in the simulation with mass $M_{200}^{\text {crit }} \geqslant 10^{14} h^{-1} \mathrm{M}_{\odot}$ by linearly interpolating the crosssection as a function of logarithmic cluster mass between the values of the ray-traced clusters.

We then compute an estimate of the differential optical depth $\partial \tau_{L / W>7.5} / \partial \chi_{\mathrm{L}}$ at the comoving line-of-sight distances $\chi_{\mathrm{L}}=\chi\left(z_{\mathrm{L}}\right)$ of these three redshifts $z_{\mathrm{L}}$ by

$$
\frac{\partial \tau_{L / W>7.5}}{\partial \chi_{\mathrm{L}}}=\chi_{\mathrm{L}}^{2} \frac{\sum_{i} \sigma_{L / W>7.5, i}}{V_{\mathrm{box}}}
$$

where the $\sigma_{L / W>7.5, i}$ are assumed to be in steradians and the summation goes over all clusters with $M_{200}^{\text {crit }} \geqslant$ $10^{14} h^{-1} \mathrm{M}_{\odot}$ found in the simulation output at redshift $z_{\mathrm{L}}$.

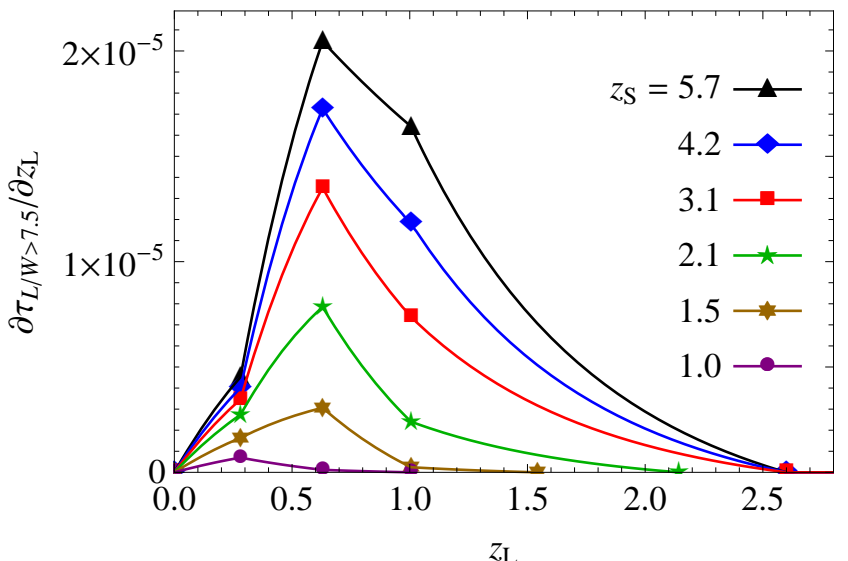

Figure 7. Differential optical depths $\partial \tau_{L / W>7.5} / \partial z_{\mathrm{L}}$ for $\operatorname{arcs}$ with length-to-width ratio $L / W>7.5$ as a function of lens reshift $z_{\mathrm{L}}$ for various source redshifts $z_{\mathrm{S}}$. The values obtained from full ray-tracing including the stellar matter and considering only arcs within 5 Einstein radii of the lens cluster are shown as symbols. The lines indicate the differential optical depths obtained from linear interpolation in the comoving line-of-sight distance $\chi_{\mathrm{L}}$.

$7 V_{\mathrm{box}}=\left(500 h^{-1} \mathrm{Mpc}\right)^{3}$ is the comoving volume of the simulation box.

Furthermore, we assume that $\partial \tau_{L / W>7.5} / \partial \chi_{\mathrm{L}}$ vanishes for $z_{\mathrm{L}}=0, z_{\mathrm{L}} \geqslant z_{\mathrm{S}}$, and $z_{\mathrm{L}}>2.6$ (there are no clusters with $M_{200}^{\text {crit }} \geqslant 10^{14} h^{-1} \mathrm{M}_{\odot}$ for $\left.z_{\mathrm{L}}>2.6\right)$. The contribution from other redshifts is then calculated by linear interpolation in $\chi_{\mathrm{L}}$. The resulting differential optical depths are illustrated for all considered source redshifts in Fig. 7. We show them as a function of the lens redshift $z_{\mathrm{L}}$ to make them more easily comparable to other works.

The optical depth $\tau_{L / W>7.5}$ is obtained by integrating $\partial \tau_{L / W>7.5} / \partial \chi_{\mathrm{L}}$ between $\chi_{\mathrm{L}}=0$ and $\chi_{\mathrm{L}}=\chi\left(z_{\mathrm{S}}\right)$. Similarly, we calculate the optical depth $\tau_{L / W>10}$ for arcs with $L / W>10$. The optical depths resulting from full raytracing including the stellar matter in galaxies is compared to the estimates based on cluster-only ray-tracing with and without stellar matter in Fig. 8

The qualitative behaviour of $\tau_{L / W>7.5}$ and $\tau_{L / W>10}$ is very similar. The additional matter along the line-of-sight increases the optical depths by $10 \%$ for $z_{\mathrm{S}}=2.1$ and by $25 \%$ for $z_{\mathrm{S}}=5.7$. Hence, projection effects are more important for higher source redshifts. The stars in galaxies enhance the optical depths by a factor two for $z_{\mathrm{S}}=1$ and cause an increase by $25 \%$ for sources at $z_{\mathrm{S}}=5.7$. Hence, the stellar matter is more important for lower source redshifts. Together, the stars and the additional matter along the line-of-sight increase the optical depth by a factor 1.5 to 2 .

For comparison with Fedeli et al. (2008), we compute

7 Note that the contribution of low-mass clusters to the optical depth is more important for high redshift sources. Our data suggest that for halos with $M_{200}^{\text {crit }}<10^{14} h^{-1} \mathrm{M}_{\odot}$, it becomes nonnegligible for $z_{\mathrm{S}} \geqslant 2.1$. This contribution is not included in this analysis. On the other hand, it is likely to strongly depend on the exact selection criteria for arcs. For example requiring some minimum value for the image separation would largely suppress it. 


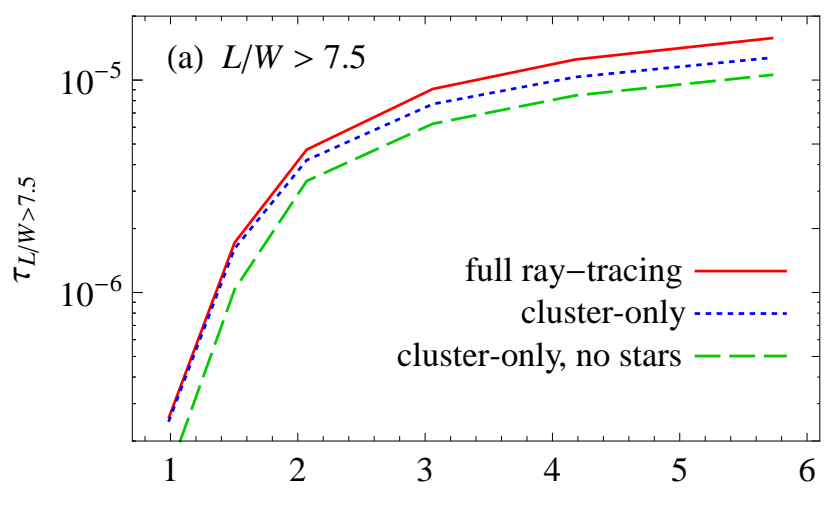

$z_{\mathrm{S}}$

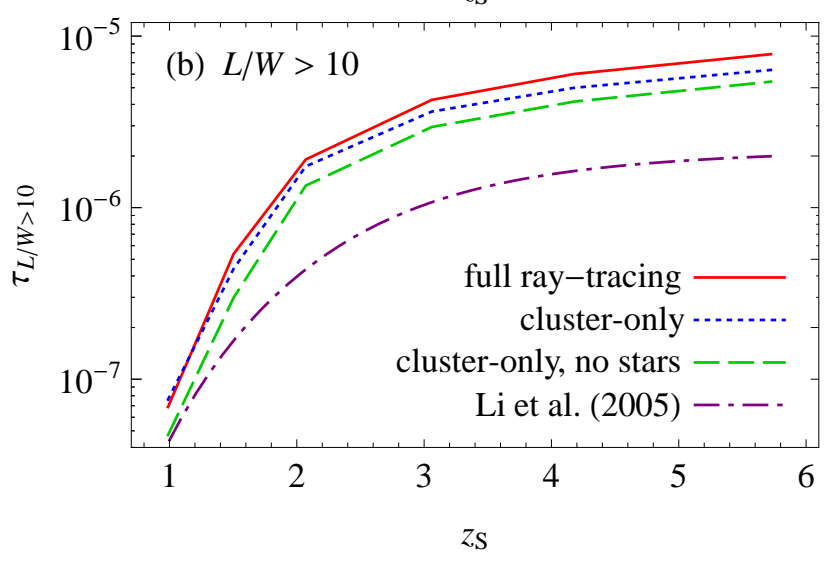

Figure 8. Optical depths $\tau_{L / W>7.5} \quad((\mathrm{a})$, top panel) and $\tau_{L / W>10}((\mathrm{~b})$, bottom panel), for arcs with length-to-width ratio $L / W>7.5$ and $L / W>10$ as a function of source redshift $z_{\mathrm{S}}$. Shown are the values for full ray-tracing including the stellar matter (red solid lines), for cluster-only ray-tracing with stars (blue dotted lines), and for cluster-only ray-tracing without stars (green dashed lines). Only arcs within 5 Einstein radii were included. Also shown is $\tau_{L / W>10}$ computed by Li et al. (2005) for sources with $1^{\prime \prime}$ effective diameter (purple dash-dotted line).

average optical depths

$$
\bar{\tau}_{L / W>7.5}=\int \mathrm{d} z_{\mathrm{S}} p_{\mathrm{S}}\left(z_{\mathrm{S}}\right) \tau_{L / W>7.5}\left(z_{\mathrm{S}}\right)
$$

using the source redshift distribution (Smail et al. 1995)

$$
p_{\mathrm{S}}\left(z_{\mathrm{S}}\right)=\frac{3}{2} z_{\mathrm{S}}^{2} \exp \left(-z_{\mathrm{S}}^{3 / 2}\right) \text {. }
$$

The resulting values $\bar{\tau}_{L / W>7.5}=2.5 \times 10^{-6}$ for full raytracing and $\bar{\tau}_{L / W>7.5}=1.7 \times 10^{-6}$ for cluster-only raytracing without stars are 4 to 6 times smaller than the optical depths of Fedeli et al. (2008). Part of the discrepancy may be due to the fact that we assumed larger source radii at low redshifts. In addition, there may be some difference in the lensing properties between the simulated cluster population used in this work and the analytic cluster population model employed there. In particular, cluster mergers are accounted for in different ways. In our simulations, cluster mergers naturally occur. However, we might be limited by small-number statistics. Fedeli et al. (2008), on the other hand, explicitly include cluster mergers, assuming that they proceed at the gravitational free-fall timescale and can be represented by two NFW halos approaching at constant ve- locity. Without the merger contribution their optical depths would be $\sim 3$ times smaller and agree much better with our results. However, Hennawi et al. (2007) question that mergers can significantly boost optical depths for giant arcs. Considering all these points, it seems likely that at least part of the discrepancy between our results and those of Fedeli et al. $(2008)$ is related to the way cluster mergers are accounted for, i. e. the importance of merger boosting of strong lensing cross-sections may be overestimated in Fedeli et al. (2008) and/or our selected cluster sample may still be too small to fully account for strong boosts during rare major cluster mergers.

In Fig. 8(b), we compare our optical depths for arcs with $L / W>10$ to those obtained by Li et al. (2005). The optical depths are very similar for sources at $z_{\mathrm{S}}=1$. However, our optical depths are 3 to 4 times larger for $z_{\mathrm{S}} \geqslant 2.1$. The fact that we used smaller source radii at higher redshifts accounts only for an increase of about $50 \%$ (Li et al. 2005). A reason for the remaining discrepancy could be the significantly better spatial resolution of the Millennium Simulation compared to the simulations used by $\mathrm{Li}$ et al. (2005), which is likely to boost the lensing efficiencies of poor clusters, whose contribution to the optical depth is most important at high source redshift. Another reason may be the differences in the assumed cosmology.

Admittedly, there is some uncertainty in our optical depth estimates due to the rather rough sampling of the cluster population, especially in cluster redshift. This may contribute to some of the discrepancies mentioned above. However, as our results deviate from those of Fedeli et al. (2008) and Li et al. (2005) in opposite directions, it is certainly not the only cause. Also the relative changes in the optical depths due to including secondary structures along the line-of-sight and the stellar components of galaxies should be robust as sparse sampling will affect all results in a similar way.

\subsection{Einstein radii}

It is straightforward to define an Einstein radius for a spherically symmetric lens, but there are several ways to generalise the concept of an Einstein radius to realistic, i.e. nonspherical, cluster lenses. In Eq. (1), we defined the Einstein radius $\theta_{\mathrm{E}}$ of a cluster via the solid angle enclosed by its outer critical curve. Another common definition is the radius $\theta_{\mathrm{E}}^{\prime}$ of a circular region around the cluster centre having a mean projected surface mass density equal to the critical density for strong lensing. In the following, we compare the two definitions.

We computed $\theta_{\mathrm{E}}^{\prime}$, which we conveniently obtain from each cluster's projected surface mass density profile, for the 750 most massive clusters in the Millennium simulation. Furthermore we used single-plane ray tracing 8 to calculate $\theta_{\mathrm{E}}$ for the 100 most massive Millennium halos and all additional lower mass clusters available in the cluster sample that we used in the previous sections. Comparing the results for all clusters for which both values were derived, we find that

8 We are most interested in large Einstein radii produced by massive clusters, which are not strongly affected by additional structures along the line-of-sight. 


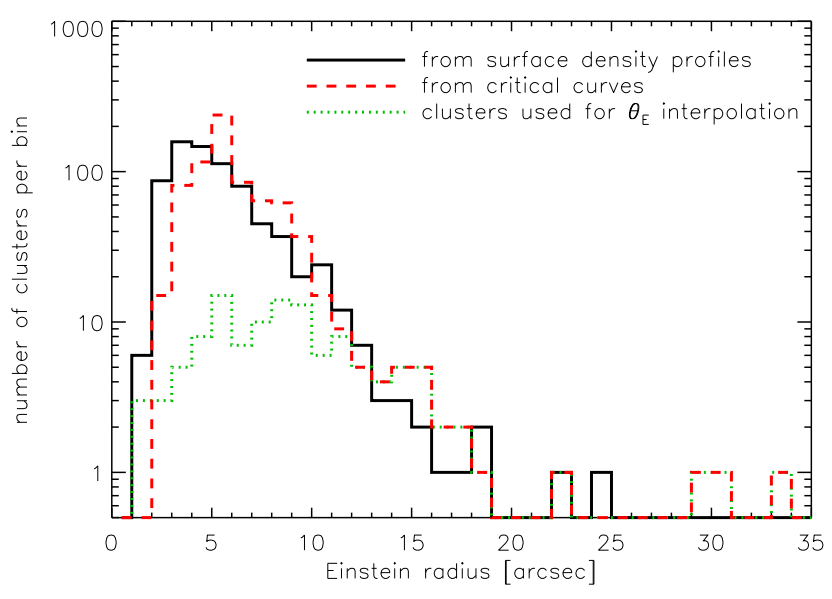

Figure 9. Distribution of Einstein radii of the 750 most massive Millennium clusters at $z_{\mathrm{L}}=0.28$ and sources at $z_{\mathrm{S}}=2.1$. Distributions are given for the Einstein radius $\theta_{\mathrm{E}}^{\prime}$, derived from surface density profiles (solid line), and $\theta_{\mathrm{E}}$, derived from the critical curves (dashed line). Also shown is the $\theta_{\mathrm{E}}$-distribution of the clusters the $\theta_{\mathrm{E}}$-interpolation was based on (dotted line).

the Einstein radii defined via the critical curve are on average $10-30 \%$ larger (depending on cluster mass) than the radii obtained from the surface density profiles. For example, the mean and $1 \sigma$-scatter of the ratios $\theta_{\mathrm{E}} / \theta_{\mathrm{E}}^{\prime}$ for sources at $z_{\mathrm{S}}=2.1$ and clusters at redshift $z_{\mathrm{L}}=0.28$ in mass bins (a)-(e) are $1.12 \pm 0.12,1.15 \pm 0.18,1.18 \pm 0.18,1.32 \pm 0.11$, and 1.28 , respectively. 9 The scatter is mostly uniform with only very few large outliers, which are related to merger processes.

The difference between $\theta_{\mathrm{E}}^{\prime}$ and $\theta_{\mathrm{E}}$ can also be seen in Fig. 9. which shows the distribution of Einstein radii $\theta_{\mathrm{E}}^{\prime}$ and $\theta_{\mathrm{E}}$ of the 750 most massive clusters in the Millennium simulation at $z_{\mathrm{L}}=0.28$, i.e. for all clusters at that redshift with $M_{200}^{\text {crit }}>1.47 \times 10^{14} h^{-1} \mathrm{M}_{\odot}$. The $\theta_{\mathrm{E}}$-distribution is based on 120 clusters for which $\theta_{\mathrm{E}}$ was calculated employing singleplane ray tracing. All other clusters were assigned a $\theta_{\mathrm{E}}$-value by linear interpolation in cluster mass. Obviously, the $\theta_{\mathrm{E}^{-}}$ distribution is shifted towards larger values compared to the $\theta_{\mathrm{E}}^{\prime}$-distribution. The Einstein radius distribution functions shown here should also be valuable for calibrating semianalytical model predictions of the Einstein radius distribution such as those in Oguri and Blandford (2009), who suggested they might be used as cosmological probes.

\subsection{Radial distribution of giant arcs}

Here, we study at what cluster-centric radii long thin arcs are most likely to be found. For a perfectly spherical symmetric halo, the longest arcs are expected to appear close to the Einstein radius. However, for a realistic cluster, this is, in general, not the case. Even prominent giant arcs that consist of multiple merged images of a background galaxy and thus cross the tangential critical curve may be significantly outside the Einstein radius due to the critical curve's ellipticity. Arcs that do not consist of multiple images can be found at even larger radii. This can be seen in the left panels

9 No scatter is given for mass bin (e) as it contains only one object.

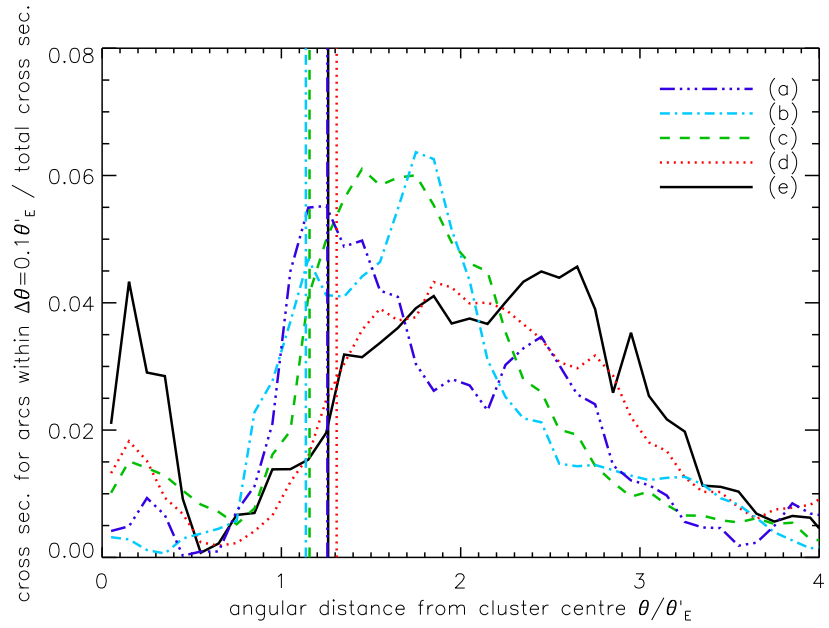

Figure 10. Radial distribution of arcs with $L / W>7.5$ for sources at redshift $z_{\mathrm{S}}=2.1$ and clusters in mass bins (a)-(e) at redshift $z_{\mathrm{L}}=0.28$. The angular distance is given in units of the Einstein-radius $\theta_{\mathrm{E}}^{\prime}$, which is calculated for each cluster from a projected surface mass density profile. The vertical lines indicate the Einstein-radii derived from the areas enclosed by the clusters' critical curves. Full ray-tracing was used.

of Fig. 3 which show that the centres of arcs with length to width ratios exceeding 7.5 are typically located outside the most distant parts of the tangential critical curve.

We investigate the radial distribution of arcs in more detail by calculating cross-sections for arcs with $L / W>7.5$ as a function of radial distance in specific radial bins. In Fig. 10, the results are shown for the five cluster mass bins listed in Table 1. In total 39 clusters at redshift $z_{\mathrm{L}}=0.28$ and full ray-tracing were used in this analysis. The clustercentric angular distance is given in units of the Einstein radius $\theta_{\mathrm{E}}^{\prime}$. All curves were normalized by the total stronglensing cross-section for arcs at all radii. Also indicated are the mean Einstein radii obtained from the critical curves of the clusters in each bin 10

One can clearly distinguish the contribution of radial and tangential arcs to the radial distribution of arcs. The former are typically found at $\theta<0.6 \theta_{\mathrm{E}}^{\prime}$. In contrast, the radial distribution of tangential arcs is very broad, and extends from $\sim 0.6 \theta_{\mathrm{E}}^{\prime}$ out to $\sim 4 \theta_{\mathrm{E}}^{\prime}$. Using a larger threshold value for the length-to-width ratio makes the distribution only slightly narrower. For $L / W>10$, it still extends to values $\gtrsim 3 \theta_{\mathrm{E}}^{\prime}$. This shows that individual tangential arcs are not well suited for constraining Einstein radii, instead more detailed cluster mass models are needed. It is, thus, important to keep the broad distribution of tangential arcs in cluster-centric radius in mind when interpreting stronglensing observations of galaxy clusters.

\section{SUMMARY AND CONCLUSIONS}

We have used ray-tracing through the Millennium simulation to study how secondary matter structures along the

10 The indicated $\theta_{\mathrm{E}} / \theta_{\mathrm{E}}^{\prime}$ values differ slightly from the numerical values quoted previously in the text as full ray-tracing and a smaller cluster sample were used here. 
line-of-sight affect strong cluster lensing, in particular the cross-section for giant arcs. We also investigated the impact of the stellar components of both cluster and interloping galaxies on cluster lensing efficiencies. Furthermore, we explored the distribution of the cluster Einstein radii and the radial distribution of giant arcs.

We performed ray-tracing along backward light cones using a multiple-lens-plane algorithm that takes light deflection by all matter structures between source and observer into account. We also performed single-lens-plane raytracing simulations that either considered only light deflection by the cluster itself or by all the matter contained in the same lens plane as the cluster. Thus in the last case, the effects of correlated structures were included while independent matter structures at different redshifts were not.

Comparing the results obtained by the different kinds of ray-tracing allows us to clearly pin down the impact of additional matter structures along the line-of-sight:

- The strong-lensing efficiency of clusters increases when including the effects of additional structures along the lineof-sight.

- The enhancement is larger for higher source redshifts.

- The enhancement is mainly due to structures along the line-of-sight that are not correlated with the lens.

- For individual clusters, boosts of the strong-lensing cross-section of up to $50 \%$ occur frequently for sources at redshift 3 and higher.

- The strong cluster lensing optical depth increases by $10 \%$ for $z_{\mathrm{S}}=2.1$ and by $25 \%$ for $z_{\mathrm{S}}=5.7$ when including the effects of additional structures along the line-of-sight.

Using the Millennium simulation's semi-analytic galaxy catalogue, we also investigated how the stellar components of galaxies affect the lensing properties of clusters. Comparing the results obtained when neglecting and including light deflection by the stellar components of cluster and interloping galaxies, we find:

- The strong-lensing properties of massive clusters are only mildly affected by stellar matter.

- The lensing cross-sections of less massive clusters can be significantly boosted by cluster galaxies.

- The enhancement due to the stellar matter is largest for low cluster and source redshift.

- Interloping galaxies do not affect the cross-section significantly.

- Including stellar matter boosts the strong-lensing optical depth by a factor of 2 for $z_{\mathrm{S}}=1$ and by $25 \%$ for $z_{\mathrm{S}}=5.7$.

Together, the stars and the additional matter along the line-of-sight increase the strong-lensing optical depth by a factor of 1.5 to 2 , depending on source redshift.

Furthermore, we computed the distribution of Einstein radii using two different definitions of the Einstein radius. We also determined the radial distribution of arcs compared to the Einstein radius of the lensing cluster. We obtain the following results:

- The Einstein radii defined via surface mass density profiles and via the area enclosed by the critical curve differ by $10 \%$ to $30 \%$.

- The contributions of radial and tangential arcs to the distribution of arcs in cluster-centric radius can be clearly distinguished.

- The radial distribution of tangential arcs is very broad and extends out to several Einstein radii. Thus, individual arcs are not well suited for constraining Einstein radii, instead, more detailed mass models are needed.

The work presented here shows that both additional structures along the line-of-sight and stellar matter in cluster galaxies do affect the strong-lensing properties of galaxy clusters significantly. Since these effects can boost cluster strong-lensing cross-sections by factors $\gtrsim 2$, they need to be taken into account when using cosmological simulations to derive predictions for the giant arc abundance and comparing them to observations. Just by themselves, however, they seem to be too small to fully account for the reported discrepancy between predicted and observed giant arc abundance in a low- $\sigma_{8}$ universe (see, e.g., Fedeli et al. 2008). Thus, other ingredients appear to be needed in addition to solve the arc statistics problem.

An obvious extension to the work presented here is the calculation of the expected number of giant arcs in surveys with various selection functions. This will require an accurate knowledge of the source redshift and luminosity distribution, in particular at high redshifts, as well as a proper treatment of magnification bias.

The discrepancies between our results for the optical depths and those based on lower-resolution simulations clearly show the need for structure formation simulations that accurately resolve the inner regions of clusters on scales of a few kpc. On these scales, the complex interplay between dark matter, gas, and stars is expected to strongly influence the matter distribution. Advances in structure formation simulations that incorporate physical processes such as cooling, star formation, and feedback will help to quantify the impact of baryonic physics on the giant arc abundance more accurately.

\section{ACKNOWLEDGMENTS}

We would like to thank Simon White, Matthias Bartelmann, and Peter Schneider for many helpful discussions. We are also indebted to Massimo Meneghetti for access to a previous version of the code used for placing sources and finding the parameters of their images. Furthermore, we would like to express our gratitude to Volker Springel, Simon White and Gabriella De Lucia for providing us access to the Millennium simulation and the semi-analytic galaxy catalogue. This work was supported in parts by the DFG within the Priority Programme 1177 under the projects SCHN 342/6 and WH $6 / 3$.

\section{REFERENCES}

Bartelmann M., Weiss A., 1994, A\&A, 287, 1

Bartelmann M., Steinmetz M., Weiss A., 1995, A\&A, 297, 1

Bartelmann M., Huss A., Colberg J.M., Jenkins A., Pearce F.R., 1998, A\&A, 330, 1

Dalal N., Holder G., Hennawi J.F., 2004, ApJ, 609, 50

De Lucia G., Blaizot J., 2007, MNRAS, 375, 2 
de Vaucouleurs G., 1948, Annales d'Astrophysique, 11, 247

Fedeli C., Bartelmann M., Meneghetti M., Moscardini L., 2008, A\&A, 486, 35

Ferguson H.C., et al., 2004, ApJ, 600, L107

Flores R.A., Maller A.H., Primack J.R., 2000, ApJ, 535, 555

Hattori M., Watanabe K., Yamashita K., 1997, A\&A, 319, 764

Hennawi J.F., Dalal N., Bode P., Ostriker J.P., 2007, ApJ, 654,714

Hilbert S., White S.D.M., Hartlap J., Schneider P., 2007, MNRAS, 382, 121

Hilbert S., Hartlap J., White S.D.M., Schneider P., 2008a, ArXiv e-prints: 0809.5035

Hilbert S., White S.D.M., Hartlap J., Schneider P., 2008b, MNRAS, 386, 1845

Komatsu E., et al., 2009, ApJS, 180, 330

Li G.L., Mao S., Jing Y.P., Bartelmann M., Kang X., Meneghetti M., 2005, ApJ, 635, 795

Meneghetti M., Bolzonella M., Bartelmann M., Moscardini L., Tormen G., 2000, MNRAS, 314, 338

Meneghetti M., Bartelmann M., Moscardini L., 2003, MNRAS, 346, 67

Meneghetti M., Argazzi R., Pace F., Moscardini L., Dolag K., Bartelmann M., Li G., Oguri M., 2007, A\&A, 461, 25 Miralda-Escude J., 1993a, ApJ, 403, 497

Miralda-Escude J., 1993b, ApJ, 403, 509

Molikawa K., Hattori M., Kneib J.P., Yamashita K., 1999, A\&A, 351, 413

Oguri M., Blandford R.D., 2009, MNRAS, 392, 930

Oguri M., Lee J., Suto Y., 2003, ApJ, 599, 7

Puchwein E., Bartelmann M., Dolag K., Meneghetti M., 2005, A\&A, 442, 405

Rozo E., Nagai D., Keeton C., Kravtsov A., 2008, ApJ, 687, 22

Smail I., Hogg D.W., Yan L., Cohen J.G., 1995, ApJ, 449, L105+

Springel V., 2005, MNRAS, 364, 1105

Springel V., et al., 2005, Nature, 435, 629

Torri E., Meneghetti M., Bartelmann M., Moscardini L., Rasia E., Tormen G., 2004, MNRAS, 349, 476

Wambsganss J., Bode P., Ostriker J.P., 2004, ApJ, 606, L93

Wambsganss J., Bode P., Ostriker J.P., 2005, ApJ, 635, L1

Wambsganss J., Ostriker J.P., Bode P., 2008, ApJ, 676, 753

Wu X.P., Mao S., 1996, ApJ, 463, 404 